\title{
Cross Cultural Variation in Emotion Regulation: A Systematic Review
}

\author{
Nosheen Ramzan, ${ }^{1}$ Naumana Amjad ${ }^{2}$
}

\section{Abstract}

A systematic review of literature on emotion regulation is carried out with the aim to identify, analyze and compare the commonly used emotion regulatory strategies (cognitive reappraisal and expressive suppression) in individualistic and collectivistic cultures including Pakistan and to study outcomes of different emotion regulation strategies in culture specific context. A systematic search has been conducted for required articles which have been published between 1990 and 2015. Only those studies have been included in the review which reported either cognitive reappraisal or expressive suppression in their findings. Review indicates that individualistic cultures prefer emotional expression to regulate emotions while collectivistic cultures, such as in Pakistan, focus more on expressive suppression. Emotional suppression can cause mental disease, physiological illness, and poor social and psychological adjustment. To sum up, culture predicts whether individuals are motivated to express or suppress their emotions. Collectivist culture encourages greatest control on emotion expression

\footnotetext{
${ }^{1} \mathrm{PhD}$ Scholar (Psychology), Institute of Applied Psychology, University of the Punjab, Lahore, Pakistan.

${ }^{2}$ Associate Professor, Institute of Applied Psychology, University of the Punjab, Lahore, Pakistan.
}

Date of Submission: 10-03-2016

Date of Acceptance for publication: 01-02-2017

Conflict of Interest: None

Funding Source: None

\section{Contribution}

All Authors have contributed in Study Design, Data Collection, Data Analysis, Data Interpretation, Manuscript Writing and Approval. while interacting with family and friends with more emphasis on maintaining social harmony. On the other hand, individualistic culture endorses comparatively less expression of negative emotions especially towards strangers. Adjusting one's emotion to the social environment is more important in cultural context.

Key words: Cognitive appraisal; Expressive suppression; Individualistic; Collectivistic.

\section{Introduction}

Emotions are central components of human experiences, which are understood as outcomes of emotion regulation. ${ }^{1}$ Emotional experiences may be similar across different cultures. ${ }^{2}$ Peeping in to the background, the concept about emotion regulation has been conceived by researchers in different ways. It refers to all means, intrinsic or extrinsic, of appraising and adjusting emotion reactions in order to establish and maintain good relationships. ${ }^{3}$ Gross, $(2001)^{4}$ defines emotion regulation as a conscious and non-conscious strategies employed to increase, maintain, or decrease one or more components of an emotional response. Gross, Richards, and John, (2006) ${ }^{5}$ defined emotion regulation as 'all the processes that help to attain culturally appropriate (or functional) emotional experiences'. However, strategies employed to regulate emotional experiences may vary due to variations in cross cultural orientations such as in individualistic (independent) versus collectivistic (interdependent) cultures For example, passive emotion regulation strategies may be compensatory strategies in some culture but optimal solutions in. ${ }^{6}$

Two frequently used emotion regulatory strategies cognitive reappraisal (antecedent-focused) and expressive suppression (response-focused) not only determine the type of emotion, but also decide when and 
how to show that emotion. ${ }^{7}$ Cognitive reappraisal comes early in emotion-generative process. It is an attempt to reinterpret an emotion-eliciting situation in a way that alters its emotional impact. ${ }^{8}$ Expressive suppression (response-focused) comes late in the emotion-generative process. It is an attempt to hide, suppress or reduce ongoing / already generated emotion and thus modifies behavioral aspect of emotional response. ${ }^{9}$ These strategies are important because they take place for many reasons: such as it helps in feeling good, to express one's feelings out and in pursuing different goals which are interpersonal in nature. ${ }^{10}$

The process of emotion regulation is multi-faceted. It is not the emotion regulation alone which causes the differences in emotions. Gross (1998, p.283) ${ }^{1}$ proposed two types of processes namely: Firstly, Situation selection involves approaching or avoiding certain people, places, or objects in order to regulate emotions. Second is Appraisal which is used to reduce the emotional intensity by changing the meaning of an upcoming event. It is related to cognitive change and usually used after an emotion has occurred. ${ }^{11}$ Reappraisal has been shown to effectively modify (increase or reduce) subjective emotional responding. ${ }^{12}$ Regulation more often takes the shape of re-appraisal, when other persons/conditions provide different perspective on the emotion situation. ${ }^{2}$

What are possible explanations for cross-cultural variations of emotion regulation? How and why might cultures differ in their use of emotion reappraisal and emotion suppression? The key aspects of these variations have been mentioned as cultural orientation towards collectivism or individualism and dialectical beliefs. ${ }^{13}$ Individualistic cultures view individuals as self directed and self-sufficient beings that they do not need to be tie strongly to any family or group. Thus, individuals are encouraged to express themselves and to develop their own individuality. ${ }^{14}$ People oriented towards individualism are more likely to perceive emotion suppression as compromising their sense of control, less satisfactory, and stress inducing. The suppression in return results in poor adjustments, both psychologically and physically. ${ }^{15}$ Collectivism on the other hand, refers to the way in which each member of a group is strongly interconnected with one another and they are assumed to be loyal with each other throughout their life. The collectivistic culture emphasizes on minimizing individuality by defining preset goals, attitude and behavior from birth to onwards. The collectivistic culture endorses the emotion regulation strategy as it is adaptive in fulfilling pro-social goals.

Dialectical Beliefs or cultural scripts play an important role in emotion regulation strategies. In Eastern collectivist culture, emphasis is on "middle way". ${ }^{16}$ Asian collectivistic values are more accustomed to moderated emotions, more tolerant of negative emotions and more flexible in their employment of emotion-regulation strategies. Research has shown that Western culture socialize children to regulate their positive emotions and minimize negative emotions according to their cultural script. ${ }^{17}$

It is valuable to know that how emotions are generated. Research has shown the role of brain network in emotion regulation processes. The differences in emotion regulation strategies have been studied with the help of neuro-imaging studies in response to emotional stimuli. ${ }^{18}$ Down regulation of negative emotions through cognitive reappraisal seems to be due to increased activation of medial and lateral prefrontal cortex (PFC) and simultaneous diminished activation of brain structures called amygdala and insula. Similarly, dispositional reappraisal is associated not only with activation in both dorsal anterior cingulate cortex (dACC) and dorsolateral PFC but at the same time there is decreased activity in amygdala, insula and hippocampus. ${ }^{19}$ Findings indicate that expressive suppression heavily relies on anterior insula and reduction of amygdala activation during suppression of emotions. ${ }^{20}$ Overall, it is suggested that individual differences in emotion regulation and expression suppression has been associated with higher amygdala activation.

\section{Objective}

To examine and compare the commonly used emotion regulation strategies in individualistic and collectivistic cultures including Pakistan and possible outcomes of using these strategies in culture specific context.

\section{Method}

\section{Inclusion Criteria for Study Selection}

A systematic search has been conducted for required articles which have been published between 1990 and 2015. Little work was done on psychology of emotion regulation prior to the mid 1985s. Bulk of the work on emotion regulation strategies has been done after 1990s. Only those studies have been included in the review which reported either cognitive reappraisal or 
expressive suppression in their findings. Moreover, for the study of emotional responses, studies recruiting adult and child population belonging to various demographic groups (such as college / university students or clinical patients) have been considered in the review. The included studies exhibited at least two cross-sectional groups of different cultures or same culture. The emotional responses of the groups were noted either on experimental manipulation of their emotional state or on self-report questionnaire of emotion regulation.

The literature searched included a total of 42 research papers and dissertation that discussed the emotion regulation strategies and relevant outcome variables associated with emotional regulation across cultures, 31 studies including 2 dissertations meeting the inclus- ion criteria were considered suitable for systematic review.

\section{Sources Used}

Relevant articles were searched by using different sources including online central database or directory of e-thesis of national level universities and available journals of educational universities, HEC Research Repository, Medline, Pub Med, Springer and an Internet search with Google Scholar followed by manual searching. The search included the different combinations of terms including emotion regulation, cognitive appraisal, expressive suppression, individualistic, collectivistic cultures.

\section{Results and Discussion}

Table 1: Characteristics and Findings of Emotion Regulation Studies in Collectivistic Cultures.

\begin{tabular}{|c|c|c|c|c|c|}
\hline $\begin{array}{l}\text { Sr. } \\
\text { No. }\end{array}$ & $\begin{array}{c}\text { Author } \\
\text { Name/Year/Country }\end{array}$ & $\begin{array}{l}\text { Design/ } \\
\text { Method }\end{array}$ & Sample Details & Assessment Tools & Key Findings \\
\hline 1. & $\begin{array}{l}\text { Kitayama, Markus, } \\
\text { Matsumoto, and } \\
\text { Norasakkunkit, (1997) } \\
\text { Japan }\end{array}$ & Cross sectional & $\begin{array}{l}\text { College students } \\
\text { including } \\
\text { Japanese }(\mathrm{n}= \\
253) \text { and } \\
\text { Americans }(\mathrm{n}= \\
102)\end{array}$ & $\begin{array}{l}\text { Self generated } \\
\text { situations compris- } \\
\text { ing success instruct- } \\
\text { ions and failure } \\
\text { instructions related } \\
\text { to self-esteem }\end{array}$ & $\begin{array}{l}\text { Japanese culture } \\
\text { emphasizes self-criticism } \\
\text { in order to live up to the } \\
\text { expectations of others, } \\
\text { which may promote } \\
\text { calmer emotional states } \\
\text { as compared to western } \\
\text { culture who make indi- } \\
\text { vidual to feel unique and } \\
\text { happy independent self. }\end{array}$ \\
\hline 2. & $\begin{array}{l}\text { Wang, (2001) } \\
\text { China }\end{array}$ & Observational & $\begin{array}{l}25 \text { Chinese } \\
\text { mothers and } 22 \\
\text { American } \\
\text { mothers }\end{array}$ & $\begin{array}{l}\text { Self developed tasks } \\
\text { related to emotion- } \\
\text { explaining style and } \\
\text { emotion-criticizing } \\
\text { style }\end{array}$ & $\begin{array}{l}\text { American mothers } \\
\text { focused on personal and } \\
\text { non-social events as } \\
\text { compared to Chinese } \\
\text { mothers. }\end{array}$ \\
\hline 3. & $\begin{array}{l}\text { Kitayama, Mesquita, } \\
\text { and Karasawa, (2006) } \\
\text { Japan }\end{array}$ & Cross-sectional & $\begin{array}{l}\text { College students } \\
\text { including } \\
\text { Japanese }(n= \\
38) \text { and } \\
\text { American }(n= \\
49)\end{array}$ & $\begin{array}{l}\text { Four self developed } \\
\text { emotion scales, } \\
\text { defined by } \\
\text { pleasantness and } \\
\text { social orientation }\end{array}$ & $\begin{array}{l}\text { Results indicated that } \\
\text { interdependent cultures } \\
\text { like Japan were more } \\
\text { likely to express } \\
\text { engaging emotions such } \\
\text { as friendliness and guilt } \\
\text { whereas independent } \\
\text { culture like North } \\
\text { American culture fosters } \\
\text { disengaging emotions } \\
\text { such as pride and anger. }\end{array}$ \\
\hline 4. & $\begin{array}{l}\text { Balkier, Arenas, Wolff, } \\
\text { and Barrow, (2012) } \\
\text { Turkey }\end{array}$ & Cross-sectional & $\begin{array}{l}\text { Women } \mathrm{N}= \\
108 \text { including } \\
\text { Turkish and } \\
\text { Germany } \\
\text { mentally }\end{array}$ & $\begin{array}{l}\text { Emotion Regulation } \\
\text { Questionnaire, } \\
\text { Dysfunctional } \\
\text { Attitude Scale Form } \\
\text { A, Loneliness Scale, }\end{array}$ & $\begin{array}{l}\text { Participants with a } \\
\text { Turkish cultural back- } \\
\text { ground engaged more in } \\
\text { emotion suppression than } \\
\text { German participants. It }\end{array}$ \\
\hline
\end{tabular}




\begin{tabular}{|c|c|c|c|c|c|}
\hline & & & $\begin{array}{l}\text { healthy } \\
\text { immigrants } \\
(\mathrm{n}=28), \& \\
(\mathrm{n}=26) \text {, Turkish } \\
\text { and German } \\
\text { patients with } \\
\text { Major Dep- } \\
\text { ressive Disorder } \\
(\mathrm{n}=29) \& \\
(\mathrm{n}=25) .\end{array}$ & $\begin{array}{l}\text { Positive and } \\
\text { Negative Affect } \\
\text { Schedule }\end{array}$ & $\begin{array}{l}\text { was also observed that } \\
\text { emotion suppression did } \\
\text { not lead to negative } \\
\text { outcomes in psycholo- } \\
\text { gical adjustment and } \\
\text { well-being. }\end{array}$ \\
\hline 5. & $\begin{array}{l}\text { Tahmouresi, Bender, } \\
\text { Schmitz, Baleshzar, and } \\
\text { Tuschen-Caffier, } \\
\text { (2014) } \\
\text { Iran }\end{array}$ & Cross-sectional & $\begin{array}{l}269 \text { children } \\
\text { including } \\
\text { Iranian }(\mathrm{n}=126 \\
) \text { and German (n } \\
=143)\end{array}$ & $\begin{array}{l}\text { Children's Emotion } \\
\text { Management Scale, } \\
\text { and Cognitive } \\
\text { Emotion Regulation } \\
\text { Questionnaire }\end{array}$ & $\begin{array}{l}\text { Iranian children use more } \\
\text { suppression and inhibit- } \\
\text { ion strategies than } \\
\text { German children. Iranian } \\
\text { children show stronger } \\
\text { relation between emotion } \\
\text { regulation and psycho- } \\
\text { pathology than German } \\
\text { children. }\end{array}$ \\
\hline 6. & $\begin{array}{l}\text { Savani, Morris, Naidu, } \\
\text { Kumar, andBerlia, } \\
(2011) \text {. } \\
\text { India }\end{array}$ & Cross sectional & $\begin{array}{l}\text { College students } \\
\text { including } \\
\text { Americans }(\mathrm{n}= \\
45) \text { and Indians } \\
(\mathrm{n}=40)\end{array}$ & $\begin{array}{l}\text { Self developed } \\
\text { interpersonal } \\
\text { situations of } \\
\text { affordances }\end{array}$ & $\begin{array}{l}\text { Indians reported initially } \\
\text { more adjustment to } \\
\text { situations, and Americans } \\
\text { reported situations more } \\
\text { influential in } \\
\text { experiencing emotions. }\end{array}$ \\
\hline 7. & $\begin{array}{l}\text { Morling, Kitayama, and } \\
\text { Miyamoto, (2002) } \\
\text { Japan }\end{array}$ & $\begin{array}{l}\text { Cross-sectional } \\
\text { design }\end{array}$ & $\begin{array}{l}\text { College students } \\
\text { including } \\
\text { Americans }(n= \\
\text { 100) and } \\
\text { Japanese } \\
(\mathrm{n}=96) .\end{array}$ & $\begin{array}{l}\text { Self developed } \\
\text { situational } \\
\text { questionnaire } \\
\text { comprising feelings } \\
\text { of efficacy and } \\
\text { feelings of } \\
\text { relatedness. }\end{array}$ & $\begin{array}{l}\text { Americans reported more } \\
\text { efficacies in influence } \\
\text { situations and Japanese } \\
\text { reported more relatedness } \\
\text { in adjustment situations. } \\
\text { This responding type was } \\
\text { not limited to local } \\
\text { situation with which they } \\
\text { might be particularly } \\
\text { familiar. }\end{array}$ \\
\hline 8. & $\begin{array}{l}\text { Rukmani, Sudhir, and } \\
\text { Bada Math, (2014) } \\
\text { India }\end{array}$ & Cross-sectional & $\begin{array}{l}60 \text { adults } \\
\text { including social } \\
\text { phobic patients } \\
(n=30) \text { and } \\
\text { normal healthy } \\
\text { adults }(n=30)\end{array}$ & $\begin{array}{l}\text { Cognitive emotion } \\
\text { regulation } \\
\text { questionnaire, } \\
\text { Frost's- } \\
\text { Multidimensional } \\
\text { Perfectionism Scale, } \\
\text { Ruminative } \\
\text { Response Scale }\end{array}$ & $\begin{array}{l}\text { Social phobic patients } \\
\text { tend to use less positive } \\
\text { reappraisal as an emotion } \\
\text { regulation strategy and it } \\
\text { is associated with } \\
\text { maladaptive dimension } \\
\text { of perfectionism as com- } \\
\text { pared to control group. }\end{array}$ \\
\hline 9. & $\begin{array}{l}\text { Kalantarkousheh, } \\
\text { Doostian, Godini, and } \\
\text { Aazami (2015) } \\
\text { Iran }\end{array}$ & Cross sectional & $\begin{array}{l}120 \text { addicted } \\
\text { individuals } \\
\text { taking treatment } \\
(\mathrm{n}=40) \text {, addicts } \\
\text { without } \\
\text { treatment ( } \mathrm{n}= \\
40) \text { and normal } \\
(\mathrm{n}=40)\end{array}$ & $\begin{array}{l}\text { Cognition Emotion } \\
\text { Regulation } \\
\text { Questionnaire } \\
\text { (CERQ) }\end{array}$ & $\begin{array}{l}\text { Study concluded that } \\
\text { emotional failure against } \\
\text { stressors was an } \\
\text { important factor in the } \\
\text { tendency toward } \\
\text { narcotics use. }\end{array}$ \\
\hline 10. & $\begin{array}{l}\text { Butt, Sanam and Gulzar } \\
(2012)\end{array}$ & Cross-sectional & $\begin{array}{l}100 \text { participants } \\
(50 \text { men and } 50\end{array}$ & $\begin{array}{l}\text { Heartland } \\
\text { Forgiveness }\end{array}$ & $\begin{array}{l}\text { Cognitive emotional } \\
\text { regulation was not the }\end{array}$ \\
\hline
\end{tabular}




\begin{tabular}{|c|c|c|c|c|c|}
\hline & Pakistan & & women). & $\begin{array}{l}\text { Scale,Cognitive } \\
\text { Emotional } \\
\text { Regulation } \\
\text { Questionnaire }\end{array}$ & $\begin{array}{l}\text { predictor of forgiveness } \\
\text { and men were more } \\
\text { forgiving than women } \\
\text { because they regulate } \\
\text { more their emotions. }\end{array}$ \\
\hline 11. & $\begin{array}{l}\text { Gul and Khan (2014) } \\
\text { Pakistan }\end{array}$ & Experimental & $\begin{array}{l}80 \text { participants } \\
\text { including } \\
\text { bipolar patients } \\
(n=40) \text { and } \\
\text { healthy adults } \\
(n=40)\end{array}$ & $\begin{array}{l}48 \text { photographs of } \\
\text { happy and neutral } \\
\text { faces and Emotion } \\
\text { regulation } \\
\text { questionnaire }\end{array}$ & $\begin{array}{l}\text { Bipolar patients reported } \\
\text { more frequent use of } \\
\text { emotion suppression and } \\
\text { lesser use of cognitive } \\
\text { reappraisal as emotion } \\
\text { regulation strategy. }\end{array}$ \\
\hline 12. & $\begin{array}{l}\text { Gul and Ahmad (2014) } \\
\text { Pakistan }\end{array}$ & Experimental & $\begin{array}{l}144 \text { participants } \\
\text { including } \\
\text { patients with } \\
\text { psychogenic } \\
\text { non-epileptic } \\
\text { seizures (PNES) } \\
(\mathrm{n}=72) \text { and } \\
\text { healthy control } \\
(\mathrm{n}=72)\end{array}$ & $\begin{array}{l}48 \text { photographs of } \\
\text { happy and neutral } \\
\text { faces and Emotion } \\
\text { regulation } \\
\text { questionnaire }\end{array}$ & $\begin{array}{l}\text { Patients with PNES used } \\
\text { expressive suppression to } \\
\text { regulate their emotions } \\
\text { more frequently and } \\
\text { reappraise their } \\
\text { cognitions less frequently } \\
\text { than healthy individuals. }\end{array}$ \\
\hline 13. & $\begin{array}{l}\text { Naheed, Dildar and } \\
\text { Kauser, (2014) } \\
\text { Pakistan }\end{array}$ & Cross sectional & $\begin{array}{l}210 \text { adults } \\
\text { including } \\
\text { Primary } \\
\text { hypertensive } \\
\text { group }(\mathrm{n}=70), \\
\text { Secondary } \\
\text { hypertensive } \\
\text { group }(\mathrm{n}=70) \\
\text { and normal } \\
\text { individuals }(\mathrm{n}= \\
70)\end{array}$ & $\begin{array}{l}\text { Difficulties in } \\
\text { Emotion Regulation } \\
\text { Scale, Difficulty } \\
\text { Describing Feelings } \\
\text { Subscale of Toronto } \\
\text { Alexithymia Scale, }\end{array}$ & $\begin{array}{l}\text { Hypertensive patients } \\
\text { reported significantly } \\
\text { more emotion regulation } \\
\text { difficulties, expression } \\
\text { suppression and } \\
\text { difficulties in describing } \\
\text { emotions than normal } \\
\text { adults. }\end{array}$ \\
\hline 14. & $\begin{array}{l}\text { Arshad and Fatima } \\
(2014) \\
\text { Pakistan }\end{array}$ & Cross sectional & $\begin{array}{l}200 \text { marital } \\
\text { couples }\end{array}$ & $\begin{array}{l}\text { Emotion Regulation } \\
\text { Questionnaire, } \\
\text { Marital } \\
\text { Aggrandizement } \\
\text { Scale, and Marital } \\
\text { Satisfaction } \\
\text { Questionnaire for } \\
\text { older Persons }\end{array}$ & $\begin{array}{l}\text { Results revealed that } \\
\text { wives' marital } \\
\text { aggrandizement mediated } \\
\text { the relationship between } \\
\text { own and her husband's } \\
\text { cognitive appraisal and } \\
\text { marital satisfaction } \\
\text { whereas husbands' mari- } \\
\text { tal aggrandizement do } \\
\text { not mediate cognitive } \\
\text { reappraise and marital } \\
\text { satisfaction of their own } \\
\text { wives. }\end{array}$ \\
\hline
\end{tabular}

Table 2: Characteristics and findings of emotion regulation studies in individualistic cultures.

\begin{tabular}{|c|l|l|l|l|l|}
\hline $\begin{array}{c}\text { Sr. } \\
\text { No }\end{array}$ & $\begin{array}{c}|c| \\
\text { Author } \\
\text { name/year/country }\end{array}$ & $\begin{array}{c}\text { Design/ } \\
\text { Method }\end{array}$ & Sample Details & Assessment Tools & \multicolumn{1}{c|}{ Key Findings } \\
\hline 1. & $\begin{array}{l}\text { Lee, Aaker, and } \\
\text { Gardner, (2000) } \\
\text { USA }\end{array}$ & $\begin{array}{l}\text { Cross } \\
\text { sectional }\end{array}$ & $\begin{array}{l}\text { College students } \\
\text { including } \\
\text { Americans } \\
(\mathrm{n}=98) \text { and }\end{array}$ & $\begin{array}{l}\text { Positive and } \\
\text { Negative Affect } \\
\text { Schedule and } \\
\text { Importance Index }\end{array}$ & $\begin{array}{l}\text { Interdependence situations } \\
\text { have regulatory focus and } \\
\text { prevention focus motivation } \\
\text { leads to relief in the case of } \\
\text { success, and anxiety in the }\end{array}$ \\
\hline
\end{tabular}




\begin{tabular}{|c|c|c|c|c|c|}
\hline & & & $\begin{array}{l}\text { Hong Kong }(\mathrm{n}= \\
117)\end{array}$ & $\begin{array}{l}\text { \& Independence- } \\
\text { Interdependence } \\
\text { scale }\end{array}$ & case of failure \\
\hline 2. & $\begin{array}{l}\text { Schmidt, Tinti, } \\
\text { Levine and Testa } \\
(2010) \\
\text { Italy }\end{array}$ & $\begin{array}{l}\text { Cross- } \\
\text { sectional }\end{array}$ & $\begin{array}{l}\text { Italian students } \\
(\mathrm{N}=610)\end{array}$ & $\begin{array}{l}\text { Self developed } \\
\text { questionnaire } \\
\text { related to different } \\
\text { emotions and } \\
\text { emotion regulation } \\
\text { strategies }\end{array}$ & $\begin{array}{l}\text { Experienced emotions were } \\
\text { related to different appraisal } \\
\text { profiles like feelings of } \\
\text { anxiety/fear and } \\
\text { frustration/powerlessness. }\end{array}$ \\
\hline 3. & $\begin{array}{l}\text { Trommsdorff and } \\
\text { Friedlmeier, (2010) } \\
\text { Germany }\end{array}$ & $\begin{array}{l}\text { Cohort } \\
\text { sequential }\end{array}$ & $\begin{array}{l}\text { Sample consist } \\
\text { of pre-school } \\
\text { girls and their } \\
\text { mothers from } \\
\text { Japan } \\
(n=20) \text { and } \\
\text { German } \\
(n=30)\end{array}$ & $\begin{array}{l}\text { Self developed } \\
\text { conditions related } \\
\text { to mothers' task } \\
\text { and distress-related } \\
\text { sensitivity and } \\
\text { children's distress } \\
\text { expression. }\end{array}$ & $\begin{array}{l}\text { German mothers focused on } \\
\text { the children's distress when } \\
\text { their children faced mishap } \\
\text { situations as compared to } \\
\text { Japanese mothers. No cultural } \\
\text { differences occurred for girls' } \\
\text { intensity of immediate distress } \\
\text { expression. }\end{array}$ \\
\hline 4. & $\begin{array}{l}\text { Matsumoto, (1990). } \\
\text { USA }\end{array}$ & Experimental & $\begin{array}{l}\text { College students } \\
\text { including } \\
\text { Americans } \\
(\mathrm{n}=42) \text { and } \\
\text { Japanese }(\mathrm{n}= \\
45)\end{array}$ & $\begin{array}{l}\text { Japanese and } \\
\text { Caucasian Facial } \\
\text { Expressions of } \\
\text { Emotion }\end{array}$ & $\begin{array}{l}\text { Results showed that Japanese } \\
\text { subjects as compared to } \\
\text { Americans express negative } \\
\text { emotions to out groups and } \\
\text { positive emotions to in group. }\end{array}$ \\
\hline 5. & $\begin{array}{l}\text { M atsumoto, Kasri, } \\
\text { and Kooken (1999) } \\
\text { USA }\end{array}$ & Experimental & $\begin{array}{l}\text { University } \\
\text { graduates } \\
\text { including } \\
\text { Americans } \\
(\mathrm{n}=128) \text {, and } \\
\text { Japanese }(\mathrm{n}= \\
80) .\end{array}$ & $\begin{array}{l}\text { Japanese and } \\
\text { Caucasian Facial } \\
\text { Expressions of } \\
\text { Emotion }\end{array}$ & $\begin{array}{l}\text { Americans have outward } \\
\text { tendencies of emotions display } \\
\text { and Japanese intended to have } \\
\text { internal subjective experiences } \\
\text { of suppression. }\end{array}$ \\
\hline 6. & $\begin{array}{l}\text { Matsumoto, } \\
\text { Takeuchi, Andayani, } \\
\text { Kouznetsova, and } \\
\text { Krupp, (1998). } \\
\text { USA }\end{array}$ & $\begin{array}{l}\text { Cross- } \\
\text { sectional }\end{array}$ & $\begin{array}{l}\text { Undergraduate } \\
\text { students } \\
\text { including South } \\
\text { Korean }(\mathrm{n}=71), \\
\text { Americans } \\
(\mathrm{n}=251), \\
\text { Russians }(\mathrm{n}= \\
\text { 159), and } \\
\text { Japanese }(\mathrm{n}= \\
\text { 120). }\end{array}$ & $\begin{array}{l}\text { Individualism- } \\
\text { Collectivism } \\
\text { Interpersonal } \\
\text { Assessment } \\
\text { Inventory and } \\
\text { Display Rule } \\
\text { Assessment } \\
\text { Inventory }\end{array}$ & $\begin{array}{l}\text { Russians had greatest control } \\
\text { on emotion expression while } \\
\text { interacting with family and } \\
\text { friends whereas Americans } \\
\text { had more emotional control } \\
\text { while interacting with } \\
\text { strangers. Moreover, } \\
\text { Americans and Japanese had } \\
\text { more social harmony, whereas } \\
\text { Korean and Russians preferred } \\
\text { more social identification and } \\
\text { social sharing of recognition. }\end{array}$ \\
\hline 7. & $\begin{array}{l}\text { Matsumoto, HeeYoo, } \\
\text { Fontaine and et. al., } \\
\text { (2008a) } \\
\text { USA }\end{array}$ & $\begin{array}{l}\text { Cross-- } \\
\text { sectional }\end{array}$ & $\begin{array}{l}\text { Cross national } \\
\text { sample including } \\
\text { Buddhist }(8.7 \%) \text {, } \\
\text { Catholic } \\
(21.3 \%) \text {, } \\
\text { Christians } \\
(18.6 \%) \text {, Hindu } \\
(8.5 \%) \text {, and } \\
\text { Muslims } \\
(18.7 \%) \text {. }\end{array}$ & $\begin{array}{l}\text { Display Rule } \\
\text { Assessment } \\
\text { Inventory }\end{array}$ & $\begin{array}{l}\text { Findings indicated that } \\
\text { individualistic cultures } \\
\text { endorsed relatively less } \\
\text { expressions of negative } \\
\text { emotions to out-group as } \\
\text { compared to in-group } \\
\text { interactants. They also endo- } \\
\text { rsed expressivity norms of } \\
\text { happiness and surprise to out- } \\
\text { groups relative to in-groups. }\end{array}$ \\
\hline
\end{tabular}




\begin{tabular}{|c|c|c|c|c|c|}
\hline 8. & $\begin{array}{l}\text { Matsumoto, HeeYoo, } \\
\text { Nakagawa, and et.al., } \\
\text { (2008b) } \\
\text { USA }\end{array}$ & $\begin{array}{l}\text { Cross- } \\
\text { sectional }\end{array}$ & $\begin{array}{l}\text { Sample taken } \\
\text { from } 23 \\
\text { countries } \\
\text { including } \\
\text { Buddhist (2.9\%), } \\
\text { Christian } \\
(38.4 \%) \text {, Hindu } \\
(7.7 \%) \text {, and } \\
\text { Muslims (4\%). }\end{array}$ & $\begin{array}{l}\text { Emotion } \\
\text { Regulation } \\
\text { Questionnaire }\end{array}$ & $\begin{array}{l}\text { Cultures that emphasized the } \\
\text { maintenance of social order } \\
\text { they tended to score high on } \\
\text { suppression. In contrast, } \\
\text { cultures that minimized the } \\
\text { maintenance of social order } \\
\text { and better adjustment with } \\
\text { country level scored high on } \\
\text { Suppression, and Reappraisal } \\
\text { scale. }\end{array}$ \\
\hline 9. & $\begin{array}{l}\text { Matsumoto, Nezlek, } \\
\text { andKoopmann, } \\
(2007) \\
\text { USA }\end{array}$ & $\begin{array}{l}\text { Cross- } \\
\text { sectional }\end{array}$ & $\begin{array}{l}\text { Data taken from } \\
36 \text { countries }\end{array}$ & $\begin{array}{l}\text { Expressive } \\
\text { reactions were } \\
\text { measured with self } \\
\text { developed } \\
\text { checklist of } 11 \\
\text { nonverbal } \\
\text { reactions and eight } \\
\text { verbal utterances. }\end{array}$ & $\begin{array}{l}\text { Collectivistic cultures with } \\
\text { high in long term orientations } \\
\text { were not associated with } \\
\text { greater intensity of shame or } \\
\text { guilt experiences and they tend } \\
\text { to be lass emotionally } \\
\text { expressive. }\end{array}$ \\
\hline 10. & $\begin{array}{l}\text { Eid and Diener, } \\
\text { (2001) } \\
\text { USA }\end{array}$ & $\begin{array}{l}\text { Cross- } \\
\text { sectional }\end{array}$ & $\begin{array}{l}\text { College students } \\
\text { from the United } \\
\text { States } \\
(\mathrm{n}=443), \\
\text { Australia } \\
(\mathrm{n}=292), \\
\text { Taiwan } \\
(\mathrm{n}=553) \text {, and } \\
\text { China } \\
(\mathrm{n}=558) .\end{array}$ & $\begin{array}{l}\text { Self developed } \\
\text { questionnaire for } \\
\text { life satisfaction, } \\
\text { experience of } \\
\text { emotions, and } \\
\text { personality in } \\
\text { native languages. }\end{array}$ & $\begin{array}{l}\text { American students used more } \\
\text { positive reappraisal and } \\
\text { acceptance, whereas Hong } \\
\text { Kong students differed most } \\
\text { strongly in self-blame. } \\
\text { Moreover, strong intra- } \\
\text { national variability in norms } \\
\text { for affect was present } \\
\text { particularly for collectivistic } \\
\text { nations. }\end{array}$ \\
\hline 11. & $\begin{array}{l}\text { Gross, and John } \\
\text { (2003) } \\
\text { USA }\end{array}$ & $\begin{array}{l}\text { Cross- } \\
\text { sectional }\end{array}$ & $\begin{array}{l}\text { College students } \\
\text { including Latino } \\
(\mathrm{n}=49), \\
\text { European- } \\
\text { American }(\mathrm{n}= \\
172), \text { Asian- } \\
\text { American }(\mathrm{n}= \\
131) \text { and } \\
\text { African- } \\
\text { American }(\mathrm{n}= \\
\text { 14). }\end{array}$ & $\begin{array}{l}\text { Emotion } \\
\text { Regulation } \\
\text { Questionnaire, and } \\
\text { Positive and } \\
\text { Negative Affect } \\
\text { Schedule }\end{array}$ & $\begin{array}{l}\text { Results indicated that Latino, } \\
\text { Asian, and African-American } \\
\text { participants were found to be } \\
\text { significantly more oriented } \\
\text { toward emotion suppression } \\
\text { than European-American } \\
\text { participants. }\end{array}$ \\
\hline 12. & $\begin{array}{l}\text { Soto, Perez, Kim, } \\
\text { Lee, and Minnick, } \\
\text { (2011) } \\
\text { USA }\end{array}$ & $\begin{array}{l}\text { Cross- } \\
\text { sectional }\end{array}$ & $\begin{array}{l}\text { Subsamples } \\
\text { including } \\
\text { European } \\
\text { American } \\
(\mathrm{n}=71) \text { and } \\
\text { Chinese } \\
(\mathrm{n}=100)\end{array}$ & $\begin{array}{l}\text { Emotion } \\
\text { Regulation } \\
\text { Questionnaire, } \\
\text { Satisfaction with } \\
\text { Life Scale, \& } \\
\text { Beck Depression } \\
\text { Inventory }\end{array}$ & $\begin{array}{l}\text { Results indicated that emotion } \\
\text { suppression to be associated } \\
\text { with adjustment difficulties } \\
\text { (i.e., negatively associated } \\
\text { with life satisfaction but } \\
\text { positively associated with } \\
\text { depression) among European- } \\
\text { American participants. }\end{array}$ \\
\hline 13. & $\begin{array}{l}\text { Ehring, Caffier, } \\
\text { Schnulle Silke, } \\
\text { Fischer, and Gross } \\
\text { (2010) } \\
\text { Netherlands }\end{array}$ & Experimental & $\begin{array}{l}73 \text { university } \\
\text { students } \\
\text { including } \\
\text { recovered } \\
\text { depressed group } \\
(\mathrm{n}=30), \text { never } \\
\end{array}$ & $\begin{array}{l}\text { Emotion } \\
\text { Regulation } \\
\text { Questionnaire The } \\
\text { Strategies } \\
\text { Questionnaire of } \\
\text { Emotion, \& The } \\
\end{array}$ & $\begin{array}{l}\text { Individuals vulnerable to } \\
\text { depression tend to } \\
\text { spontaneously use more } \\
\text { dysfunctional emotion } \\
\text { regulation strategies, but are as } \\
\text { effective as controls in using }\end{array}$ \\
\hline
\end{tabular}




\begin{tabular}{|c|c|c|c|c|c|}
\hline & & & $\begin{array}{l}\text { depressed group } \\
(\mathrm{n}=43) .\end{array}$ & $\begin{array}{l}\text { Positive and } \\
\text { Negative Affect } \\
\text { Schedule }\end{array}$ & $\begin{array}{l}\text { functional strategies when } \\
\text { instructions were given how to } \\
\text { regulate their emotions on } \\
\text { given task. }\end{array}$ \\
\hline 14. & $\begin{array}{l}\text { Butler, Lee, and } \\
\text { Gross, (2007) } \\
\text { USA }\end{array}$ & $\begin{array}{l}\text { Cross- } \\
\text { sectional }\end{array}$ & $\begin{array}{l}166 \text { women } \\
\text { including Asian } \\
\text { American }(\mathrm{n}= \\
63), \text { European } \\
\text { American }(\mathrm{n}= \\
74), \text { Latin } \\
\text { American }(\mathrm{n}= \\
\text { 15), and African } \\
\text { American }(\mathrm{n}= \\
14) .\end{array}$ & $\begin{array}{l}\text { Emotion } \\
\text { Regulation } \\
\text { Questionnaire, The } \\
\text { Circumplex Scale } \\
\text { of Interpersonal } \\
\text { Values, The Asian } \\
\text { values scale, \& } \\
\text { European } \\
\text { American Values } \\
\text { Scale for Asian } \\
\text { Americans. }\end{array}$ & $\begin{array}{l}\text { Results indicated that emotion } \\
\text { suppression appeared to be } \\
\text { problematic only for } \\
\text { participants oriented toward } \\
\text { individualistic culture as } \\
\text { compared to collectivism. } \\
\text { Asian women avoid using } \\
\text { suppression in situations } \\
\text { where it would have a } \\
\text { negative social impact as } \\
\text { compared to women with } \\
\text { European values. }\end{array}$ \\
\hline 15. & $\begin{array}{l}\text { Tsai, Levenson, } \\
\text { andCarstensen(2000) } \\
\text { USA }\end{array}$ & $\begin{array}{l}\text { Cross- } \\
\text { sectional }\end{array}$ & $\begin{array}{l}\text { College students } \\
\text { including } \\
\text { European } \\
\text { Americans ( } \mathrm{n}= \\
\text { 201) and Asian } \\
\text { Americans } \\
(\mathrm{n}=196)\end{array}$ & $\begin{array}{l}\text { Actual and ideal } \\
\text { affect Scale and } \\
\text { Self- Construal } \\
\text { Scale }\end{array}$ & $\begin{array}{l}\text { European American } \\
\text { individuals value high-arousal } \\
\text { positive affect. Culture } \\
\text { influences ideal affect more } \\
\text { than actual affect. Both affects } \\
\text { play a role in mental health. }\end{array}$ \\
\hline 16. & $\begin{array}{l}\text { Tahmouresi, Bender, } \\
\text { Schmitz, Baleshzar, } \\
\text { and Tuschen-Caffier, } \\
\text { (2014) } \\
\text { Germany }\end{array}$ & $\begin{array}{l}\text { Cross- } \\
\text { sectional }\end{array}$ & $\begin{array}{l}269 \text { children } \\
\text { including Iranian } \\
(\mathrm{n}=126) \text { and } \\
\text { German }(\mathrm{n}= \\
143)\end{array}$ & $\begin{array}{l}\text { Children's } \\
\text { Emotion } \\
\text { Management } \\
\text { Scale, Cognitive } \\
\text { Emotion } \\
\text { Regulation } \\
\text { Questionnaire \& } \\
\text { Youth SelfReport } \\
\text { questionnaire for } \\
\text { Internalizing \& } \\
\text { externalizing } \\
\text { symptoms. }\end{array}$ & $\begin{array}{l}\text { Iranian children use more } \\
\text { suppression and inhibition } \\
\text { strategies than German } \\
\text { children. Therefore, Iranian } \\
\text { children show stronger } \\
\text { relation between emotion } \\
\text { regulation and } \\
\text { psychopathology than German } \\
\text { children. }\end{array}$ \\
\hline 17. & $\begin{array}{l}\text { Buttler, Egloff, } \\
\text { Wilhelm, Smith, } \\
\text { Erickson and Gross, } \\
\text { (2003). } \\
\text { USA }\end{array}$ & Experimental & $\begin{array}{l}\text { Undergraduate } \\
\text { female students } \\
(\mathrm{N}=84)\end{array}$ & $\begin{array}{l}\text { Self constructed } \\
\text { Positive and } \\
\text { Negative Emotion } \\
\text { Scale and Emotion } \\
\text { Regulation } \\
\text { Questionnaire }\end{array}$ & $\begin{array}{l}\text { Expressive suppression leads } \\
\text { to decreased rapport and } \\
\text { willingness to affiliate. } \\
\text { Moreover it leads to increase } \\
\text { blood pressure. }\end{array}$ \\
\hline
\end{tabular}

\section{Measurement of Emotion Regulation Strategies}

To analyze the emotion regulation strategies in different cultures, it is important to discuss the measures used in emotion regulation studies. Most commonly used measures in majority studies are Emotion Regulation Questionnaire ${ }^{7}$ and Cognitive Emotion Regulation Questionnaire. These scales have shown good alpha reliability for reappraisal factor (.80 to .82) and suppression factor (.73 to .76). In some studies, subjective account of individuals was taken by using self- developed statements related to emotion-explaining style and emotion-criticizing style. These subjective accounts were used either in observation studies or in experimental studies with the combination of other affect related objective measures. It has also been noticed that most of the surveys conducted in both individualistic and collectivistic cultures, employed the Emotion Regulation Questionnaire. In Pakistan, Urdu version of Gross and John's Emotion Regulation Questionnaire has been used in many studies. As far as 
other cultures are concerned, researchers have used same questionnaire in their native languages.

\section{Cross-cultural Variations in Emotion Expression}

Literature has posited that cultures endorse different forms of emotion expressions which lead to different ways of emotion regulation. After reviewing the studies from both cultures included in the current systematic review, it has been observed that most studies have been conducted in the individualistic culture where emotional expression is mainly employed in emotion regulation (Figure 1).

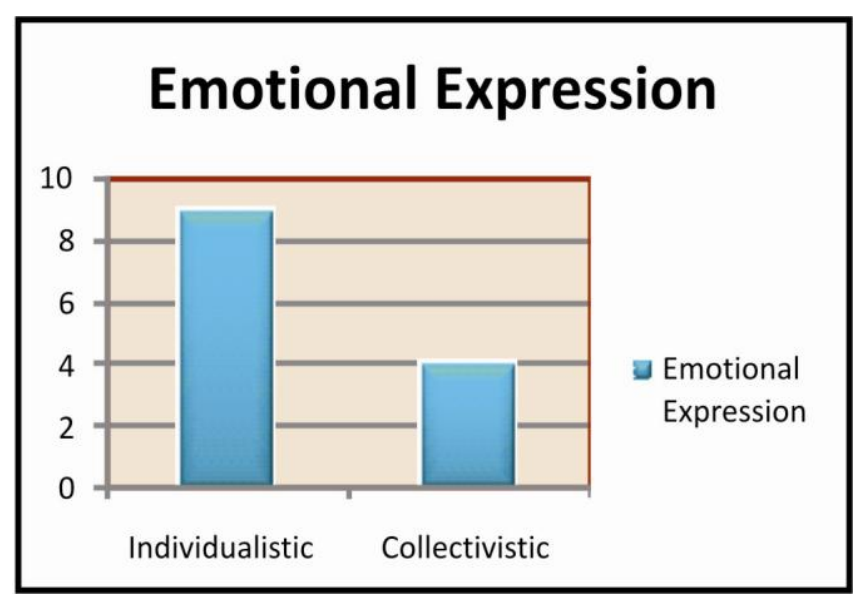

Fig. 1: Emotional Expression in Individualistic and Collectivistic Cultures.

Possible cross-cultural variations in emotion regulation have begun to emerge since Gross and John's introduction of the 'Emotion Regulation Questionnaire' (2003). It has demonstrated that different cultures hold different standards, ideals and values which led the individuals to exercise different emotion regulation strategies accordingly. More direct evidence to support individualistic and collectivistic cultural differences in emotion regulation comes from a pioneering study by Matsumoto et al. (2008a). ${ }^{21}$ These researchers examined suppression and reappraisal use, in 23 different countries by using 'Emotion Regulation Questionnaire'. They found that individuals from individualistic nations, such as the USA, Canada, and Australia, reported less use of suppression than did individuals from East Asian nations, such as China, Japan, and Korea. In contrast, they found no evidence for individualistic and collectivistic nation- level differences in reappraisal. Other studies have compared the tendencies of emotional display rules with reference to individualistic and collectivistic culture. On the whole, studies suggested that Japanese intended to show negative emotions in front of strangers ${ }^{22}$ and Americans have outward tendencies of positive emotions to strangers as compared to Japanese according to their display rules. ${ }^{23}$ Moreover, the level of emotion expressivity is more frequent in individualistic cultures. Compared to collectivistic cultures, individualistic cultures also stresses on exhibiting more amount of negative emotions within group members, but more positive emotions towards strangers. ${ }^{24}$

One study included in the review from collectivistic culture have corroborated that culture high in long term orientation tends to be less emotionally expressive. In particular, greater exposure to individualistic culture and its strong emphasis on independence of self-expression is associated with less use of suppression to maintain social harmony. For example 25 reported in their study that North American students use more positive reappraisal and acceptance, whereas Hong Kong students were found to use more of selfblame. Similarly Chinese culture also views the positive emotions as undesirable. ${ }^{26}$ Such cultural variation in positive appraisal is largely due to the freedom of emotion expression which is foster by a relevant culture among their individuals. Taken together, the results suggested that emotion expression in independent and interdependent culture varies due to their cultural orientations but these findings provide the broad overview of individualistic culture not from collectivistic culture as current review has included one study on emotion expression from collectivistic culture.

\section{Cross-cultural Variations in Emotion Suppression}

This review indicates that in collectivistic cultures (such as in Latino, Asian, and African-American set ups) participants were found to be significantly more oriented toward emotion suppression than participants of individualistic cultures (European-American set ups) (Figure 2).

Emotion regulation strategies have been investigated in areas of both intra-personal and interpersonal level in individualistic and collectivistic cultures. For example when expression suppression was investigated in collectivistic culture like Turkey and Iran in term of intrapersonal level, findings demonstrated that participants with a Turkish and Iranian cultural background engaged more in emotion suppression than German participants. Turkish are assumed to exhibit more respect for family members in order to maintain 


\section{Expressive Suppression}

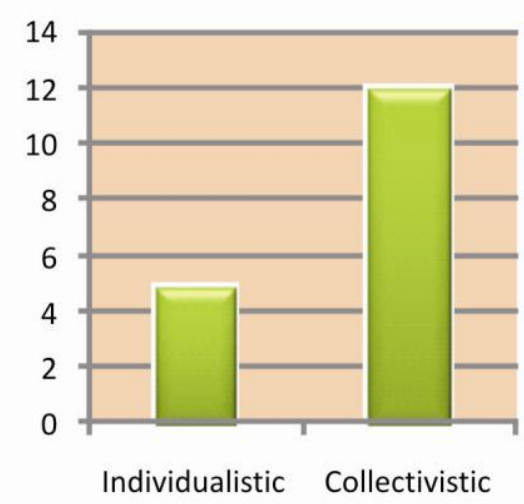

Fig. 2: Expressive Suppression in Individualistic and Collectivistic Cultures.

social harmony. ${ }^{27}, 28$

At the interpersonal level, emotion suppression has been found to have less adverse outcomes among individuals oriented toward collectivism. For example Butler, Lee, and Gross, $(2007)^{29}$ investigated the cultural variability in emotional suppression and its social consequences. Findings showed that in emotion suppression, European American participants were less responsive in their social interactions and were perceived as negative and hostile by those with whom they interacted as compared to Asian Americans. It is indicated that emotion suppression seems to be challenging only for those participants belonging to an individualistic culture, not those oriented toward collectivism. Similar results reported by. ${ }^{30}$ They found that Asian women were vigilant while using suppression in situations where it would cast negative impact on social relations as compared to those belonging to individualistic or European culture background. The possible explanation of this phenomenon of expressive suppression can be that Asian culture gives much weight on fulfilling societal demands and accommodating others that's why they practice suppression more than expression. $^{31}$

To sum up, it can be concluded that habitual suppression is self-protective in collectivistic cultures but can be problematic as it lowers receptiveness and has negative social consequences for people belonging to individualistic cultures. Culture plays an important role in using suppressive strategy, and it is not something that took place solely on individuals' own will.

\section{Sources of Cultural Variation in Emotion Regulation}

Review of the literature has also revealed that it is not the emotion regulation alone which causes the differences in emotions but there are some other underlying sources involved in emotion regulation. ${ }^{32}$ The individual tendencies, relational co-regulation, and structural condition are assumed to be the major sources of situation selection and appraisal and culture play a major role in underneath these sources.

Results of studies taken from collectivistic culture have shown that our social relations with close family members play an important role in experiencing certain type of emotions too. This phenomenon is called a co-regulation. In collectivistic cultures, mothers are assumed to shape the emotional experiences of their children by discussing and talking about those events in which other people considered more important rather talking about independent self. By doing so, mothers inculcate the interdependent nature of emotions in their children. ${ }^{33}$

In individualistic cultures, the selection of situation that occurs habitually may structure individual's emotional life in some way. It has been observed that individualistic culture structure the social life in a way that make individual feels special and unique and ultimately happy independent self-compared to collectivistic cultures like Japanese culture which emphasizes on expression suppression in social life to promote calmer emotional states at individual life. ${ }^{34}$

Researches in the current review also suggest some sources of appraisal in emotional experiences. Mainly two sources have been identified in current review: how one perceives the world based on his own beliefs and adopting other people's appraisal as a reference. This is called a 'social referencing'. The evidence for these sources of appraisal comes from the studies conducted in the individualistic culture. A study conducted $^{35}$ have indicated that when Iranian students related the exams controllable they perform well and when they rate the exams as uncontrollable then their appraisal profile was based on more frustration, anxiety and fear. The notion of social referencing has been reinforced by the study of. ${ }^{36}$ They found in their study that children interpret the events with reference to those situations which have been authenticated by parents.

Although most of studies confirm that emotion regulation strategies are influenced by individual tendencies and relation with others. But there are some evidences that indicated that emotion regulation also 
dependent in the ways, the individual's environment is structured. It is evident in the study conducted by ${ }^{37} \mathrm{In}$ this study, Indian and European students were shown a large no of interpersonal situations relevant to their culture. Results indicated that both types of situation and individual tendencies together played a crucial role in students' reactions pertaining to cultural differences.

After reviewing the studies from individualistic and collectivistic cultures, it can be concluded that people are either internally motivated in selection of situations or their viewpoint about the world will direct their appraisal while experiencing certain emotions. They usually adopt either a promotion focused motivation or a prevention focused motivation. When one promotes a desired emotion it leads to happiness and when someone is deprived of exhibiting particular emotion that causes negative feelings like depression and failure. ${ }^{38}$

\section{Outcomes of Emotion Regulation Strategies}

The current review has identified some outcomes associated with differently used emotion regulation strategies especially with expressive suppression. Cognitive reappraisal and its outcomes remain relatively constant across culture as compared to expressive suppression. Data synthesis revealed that expressive suppression has major consequences in the psychological, social and physiological domains. Each domain has been discussed separately in reference to cultural context.

\section{Psychological Consequences of Suppression}

Current review has suggested that continued emotional suppression can cause mental disease, illness, and poor psychological adjustment in both eastern and western cultures. A comparative study was conducted by, ${ }^{39}$ on European American and Chinese students. Results indicated that in European Americans emotion suppression to be associated with adjustment difficulties like poor satisfaction with life and increased level of depression. However, among Hong Kong Chinese who reported greater emotion suppression, these associations found to be absent. Similar outcomes of habitual use of suppression were reported by American students. They reported poor social adjustment, decrease in well-being and higher level of negative affect, ${ }^{40,41}$ and greater rate of psychopathology in Turkish. ${ }^{28}$

This pattern of emotional suppression is found to be similar in studies conducted in the collectivistic culture as well. Experimental investigation comparing the suppression between those having psychological disorders and healthy controls, the tendency to use suppression was higher in bipolar patients ${ }^{42}$ Social phobic patients ${ }^{43}$ and in patients of psychogenic nonepileptic seizure who used expressive suppression more frequently to regulate their emotion and reappraise their cognitions less frequently than healthy individuals. $^{44}$

\section{Social Consequences of Suppression}

The current review has suggested that expressive suppression has some utilitarian function if it is used within limited boundaries. However, an unnecessary use of expressive suppression is found to be linked with poor social interactions and decreased altruistic behavior in both cultures. ${ }^{45}$ One of the pioneer studies of emotion regulation in social domain has conducted. ${ }^{46}$ Results have indicated negative consequences of expressive suppression that in some contexts at least suppressing emotions disrupts the communication in couples. It may also limit access to new relationships and may hinder the maintenance of existing relationships. Similar findings have been reported by ${ }^{47}$ in their study they found expressive suppression was related to husbands' marital dissatisfaction and cognitive reappraisal was positively linked to marital satisfaction of wives. Expressive suppression also has been found to have some utilitarian function in some culture. For example two large scaled cross nation survey conducted by ${ }^{21}$ and. ${ }^{48}$ They included the sample from different 36 countries including varying ethnic backgrounds like Buddhism, Christian, Hindu, and Muslims. Results have indicated that suppression was associated with better adjustment in those countries who emphasized the maintenance of social order and power distance. Findings also suggested that suppression was important to maintain existing social hierarchies.

Mostly studies conducted to identify social consequences of expressive suppression have come from the individualistic cultures, so more studies are needed to confirm this pattern of social consequences in collectivistic cultures as well.

\section{Physiological Outcomes of Suppression}

Studies included in current systematic review have also indicated that poorly used emotion regulation strategies lead towards mal functioning of physiology. For example some studies conducted in collectivistic culture including Pakistan have shown that emotional failure against stressors was an important factor in the 
tendency toward narcotics use. ${ }^{49}$ In another study expressive suppression was assessed in hypertensive patients and normal adults. Results indicated that hypertensive patients reported significantly more emotion regulation difficulties, expression suppression and difficulties in describing emotions than normal adults and expressive suppression was significant factor in precipitating hypertension. ${ }^{50}$ The similar pattern of negative consequences also found in studies conducted in individualistic culture. Buttler, Egloff, Wilhelm, Smith, Erickson and Gross, $(2003)^{46}$ conducted a study on 84 female students with different cultural background to observe the physiological consequences of expressive suppression on their arterial blood pressure. Results indicated that in experimental condition, students who experienced more suppression on given task, the level of their blood pressure was higher as compared to control group.

Altogether, review of these studies suggests that excessive use of emotion suppression at interpersonal level not at intrapersonal level in collectivistic and individualistic cultures positively related to psychological maladjustment, negative social consequences and poor physical health outcomes.

\section{Conclusion}

The present review suggests that culture predicts whether individuals are motivated to express or suppress their emotions. Collectivist culture encourages greatest control on emotion expression while interacting with family and friends and lays more emphasis on maintaining social harmony. On the other hand, individualistic culture endorses comparatively less expression of negative emotions towards strangers. Individuals from individualistic cultures may benefit more from cognitive reappraisal compared to expressive suppression because adjusting one's emotion to the social environment is more important in cultural context $\mathrm{t}^{24}$ and. ${ }^{21}$ In this review it has been observed that most studies have focused in identifying expressive suppression in both cultures. The studies conducted in individualistic cultures, include large scaled surveys involving comparison of different ethnic groups using students' population. This pattern was also consistent in studies conducted in collectivistic cultures except those studies that have been done in Pakistan. Most of the studies in Pakistan have focused in identifying emotion regulation strategies in healthy versus morbid individuals. It is also evident from current review that it is not the emotion regulation alone which causes the differences in emotions but individual tendencies, relational coregulation, and structural condition are assumed to be the major sources of emotion regulation. ${ }^{32}$ But these evidences have been supported by the studies conducted in individualistic culture only. In collectivistic culture, the observed evidence for these related sources is neither prominent nor have been tested empirically. Moreover it has been observed that, researchers have used survey research design. Experimental research design was found to be least employed design especially, in collectivistic culture. Emotion regulation strategies have several outcomes; especially expressive suppression has debilitating effects in areas of psychological, social and physiological domains. The expressive suppression has both positive and negative consequences in psychological and social domains. Most of the studies have been under taken in individualistic cultures to identify inter-individuals variability in emotion regulation. However, in the collectivistic culture large scale studies are needed to endorse similar findings regarding intra-individual variability in emotion regulation strategies.

\section{Identified Directions of Future Research}

1. Present review suggests that emotion regulation has different strategies and is linked to different outcomes. Mostly researchers have measured these strategies with one single scale so far. Thus, future research should measure both strategies distinctively by developing independent measures of cognitive appraisal and expressive suppression.

2. It is important to point out that above mentioned studies have focused on two dimensions (independent and interdependent) of cultural values while recruiting two cultural groups (Asian Americans and European Americans). It will be interesting to expand this research area to other cultural values like cultural hierarchy, traditions, socioeconomic status and region.

3. The current review includes those studies which have investigated inter cultural variations in emotion regulation but intra individual differences in emotion regulation in cultural context have not been examined much. The futures researches may focus both on inter- and intra-individual differences in emotion regulation strategies.

4. The studies in current review have a limited ethnic and gender diversity. It is important to study cross cultural variations in emotion regulation strategies in more diverse population. 


\section{References}

1. Gross JJ. The emerging field of emotion regulation: An integrative review. Review of General Psycholog. 1998; 2: 271-299.

2. Leersnyder JD, Boiger M, Mesquita B. Cultural regulation of emotion: Individual, relational and structural resources. Frontiers in Psychology.2013; 4: 1-11.

3. Thompson RA. Emotional regulation: A theme in search for definition. Monographs of the Society for Research in Child Development.1994; 59: 25-52.

4. Gross JJ. Emotion regulation in adulthood: Timing is everything. Current Directions in Psychological Science. 2001; 10: 214-219.

5. Gross JJ, Richards JM, John AP. Emotion regulation in everyday life. In D. K. Snyder JA, Simpson JN. Hughes Emotion (Eds.), Regulation in Families: Pathways to Dysfunction and Health (pp 13-35). Washington. DC: APA.2006.

6. Roseman IJ, Dhawan, Rettek N, Naidu SI, Thapa K. Cultural differences and cross-cultural similarities in appraisals and emotional responses. Journal of CrossCultural Psychology.1995; 26 (1): 23-48.

7. Gross JJ, John OP. Individual differences in two emotion regulation processes: Implications for affect, relationships and well-being. Journal of Personality and Social Psychology. 2003; 85: 348-362.

8. Lazarus RS, Folkman S. Stress, appraisal and coping. New York: Springer. 1984.

9. Gross JJ, Levenson RW. Emotional suppression: physiology, self- report and expressive behavior. Journal of Personality and Social Psychology. 1993; 64: 970-986.

10. Fisher A, Manstead ASR, Evers C, Timmers M, Valk G. Motives and norms underlying emotion regulation. In P. Philippot RS. Feldman (Eds.), The regulation of emotion (pp.187-210). New Jersey: Erlbaum. Retrieved from 2004.

11. Gross JJ, Emotion Regulation: Current Status and Future Prospects. Psychological Inquiry. 2015; 26: 1-26.

12. Ochsner K, Gross J. The cognitive control of emotion. Trends in Cognitive Sciences. 2005; 9: 242-249.

13. Markus HR, Kitayama S. Culture and the self: Implications for cognition, emotion, and motivation. Psychological Review. 1991; 98 (2): 224-253.

14. Hofstede G. Culture's consequences: Comparing values, behaviors, institutions, and organizations across nations, ( $2^{\text {nd }}$ ed.). Thousand Oaks: Sage Publications. 2001.

15. Barrett LF, Mesquita B, Ochsner KN, Gross JJ. The experience of emotion. Annual Review of Psychology. 2007; 58: 373-403.

16. Miyamoto Y, Ma X. Dampening or savoring positive emotions: A dialectical cultural script guides emotion regulation. Emotion. 2011; 11 (6): 1346-1357.

17. Miller P, Wang S, Sandel, T, Cho G. Self-esteem as folk theory: A comparison of European American and
Taiwanese mothers' beliefs. Parenting: Science and Practice. 2002; 2: 209-239.

18. Ochsner K, Gross J. The cognitive control of emotion. Trends in Cognitive Sciences. 2005; 9: 242-249.

19. Hermann A, Bieber A, Keck T, Vaitl D, Stark R. Brain structural basis of cognitive reappraisal and expressive suppression. SCAN. 2014; 9: 1435-1442.

20. Goldin PR, McRae K, Ramel W, Gross JJ. The neural bases of emotion regulation: reappraisal and suppression of negative emotion. Biological Psychiatry. 2008; 63: 577-86.

21. Matsumoto D, Yoo SH, Fontaine J, Anguas-wong AM, Arriola M, Ataca B. et al.,. Mapping expressive differences around the world: The relationship between emotional display rules and individualism versus collectivism. Journal of Cross Cultural Psychology. 2008; 39: 55-74.

22. Matsumoto D. Cultural similarities and differences in display rules. Motivation and Emotion. 1990; 14 (3): 195-213.

23. Matsumoto D, Kasri F, Kooken K. American-Japanese cultural differences in judgments of expression intensity and subjective experience. Cognition and Emotion. 1999; 13 (2): 201-218.

24. Matsumoto D, Takeuchi S, Andayani S, Kouznetsova N, Krupp D. The contribution of individualism vs. collectivism to cross-national differences in display rules. Asian Journal of Social Psychology. 1998; 1: 147-165.

25. Kitayama S, Mesquita B, Karasawa M. Cultural affordances and emotional experience: Socially engaging and disengaging emotions in Japan and the United States. Journal of Personality and Social Psychology. 2006; 91 (5): 890-903.

26. Eid M, Diener E. Norms for experiencing emotions in different cultures: Inter and international differences. Journal of Personality and Social Psychology. 2001; 8: 869-885.

27. Balkir N, Arens EA, Wolff C, Barnow S. Exploring the influence of self construals on psychopathology in Turkish immigrant and German women with major depression. Psychiatrische Praxis. 2012; 40 (3): 135-145.

28. Tahmouresi N, Bender C, Schmitz J, Baleshzar A, Tuschen-Caffier B. Similarities and differences in emotion regulation and psychopathology in Iranian and German school children: A cross-cultural Study. International Journal of Preventive Medicine. 2014; 5: 52-60.

29. Butler EA, Lee TL, Gross JJ. Emotion regulation and culture: Are the social consequences of emotion suppression culture-specific? Emotion. 2007; 7 (1): 30-48.

30. Morling B, Kitayama S, Miyamoto Y. Cultural practices emphasize influence in the United States and adjustment in Japan. Personality and Social Psychology Bulletin. 2002; 28: 311-323.

31. Ehring T, Caffier BT, Schnulle J, Fischer S, Gross JJ. Emotion regulation and vulnerability to depression: 
Spontaneous versus instructed use of emotion suppression and reappraisal. Emotion. 2010; 10 (4): 563-572.

32. Ekman P. Are there basic emotions? Psychological Review.1992; 99 (3): 550-553.

33. Wang Q. Did you have fun? American and Chinese mother - child conversations about shared emotional experiences. Cognitive Development. 2001; 16: 693715.

34. Kitayama S, Matsumoto D, Markus HR, Norasakkunkit $\mathrm{V}$. Individual and collective processes in the construction of the self: self-enhancement in the US and self- criticism in Japan. Journal of Personality and Social Psychology. 1997; 72: 1245-1267.

35. Schmidt S, Tinti C, Levine LJ, Testa S. Appraisals, emotions and emotion regulation: An integrative approach. Motivation and Emotion. 2010; 34 (1): 63-72.

36. Trommsdorff G, Friedlmeier W. Preschool girls' distress and mothers' sensitivity in Japan and Germany. European Journal of Developmental Psychology. 2010; 7: 350-370.

37. Savani K, Morris MW, Naidu NVR, Kumar S, Berlia NV. Cultural conditioning: Understanding interpersonal accommodation in India and the United States in terms of the modal characteristics of interpersonal influence situations. Journal of Personality and Social Psychology. 2011; 100 (1): 84-102.

38. Lee AY, Aaker JL, Gardner WL. The pleasures and pains of distinct self-construals: The role of interdependence in regulatory focus. Journal of Personality and Social Psychology. 2000; 78: 1122-1134.

39. Soto JA, Perez CR, Kim H, Lee EA, Minnick MR. Is expressive suppression always associated with poorer psychological functioning? A cross-cultural comparison between European Americans and Hong Kong Chinese. Emotion. 2011; 11 (6): 1450-455.

40. Tsai JL, Levenson RW, Carstensen LL. Autonomic, subjective, and expressive responses to emotional films in older and younger Chinese Americans and European Americans. Psychology and aging. 2000; 15 (4): 684693.
41. Tsai JL, Miao FF, Seppala E, Fung HH, Yeung DY. Influence and adjustment goals: Sources of cultural differences in ideal affect. Journal of Personality and Social Psychology. 2007; 92: 1102-1117.

42. Gul A, Khan K. Emotion regulation strategies can predict task-switching abilities in euthymic bipolar patients. Frontier in Human Neuroscience. 2014; 8: 1-9.

43. Rukmani S, Sudhir PM, Math SB. Perfectionism, emotion regulation and their relationship to negative affect in patients with social phobia. Indian Journal of Psychological Medicine. 2014; 36 (3): 239-245.

44. Gul A, Ahmad, H. Cognitive deficits and emotion regulation strategies in patients with psychogenic non-epileptic seizures: A task-switching study .Pakistan Epilepsy and Behavior. 2014; 32: 108-113.

45. Butt MM, Sanam F, Gulzar S. Cognitive emotional regulation and forgiveness. Interdisciplinary Journal of Contemporary Research in Business. 2013; 4 (12): 6769-792.

46. Butler EA, Egloff B, Wilhelm FH, Smith NC, Erickson EA, Gross JJ. The social consequences of expressive suppression. Emotion. 2003; 3 (1): 48-67.

47. Arshad A, FatimaI. Emotion regulation, marital aggrandizement and marital satisfaction in long-wed couples. Unpublished M. Phil theses, Institute of Applied Psychology. University of the Punjab, Lahore, Pakistan. 2014.

48. Matsumoto D, Yoo SH, Nakagawa S. Culture, emotion regulation, and adjustment. Journal of Personality and Social Psychology. 2008; 94 (6): 925-937.

49. Kalantarkousheh, Doostian, Godini, Aazami. Cognitive emotion regulation strategies among regular persons and participants in Methadone or Narcotics Anonymous Treatment Programs. Studies in Social Sciences and Humanities. 2015; 2 (3): 131-138.

50. Naheed S, Dildar SR, Kausar R. Expressive suppression and emotion regulation difficulties among hypertensive patients and normal individuals. Unpublished manuscript. Institute of Applied Psychology. University of the Punjab, Lahore, Pakistan 2014. 